\title{
Developing and Simulating a Content Adaptation Tool for Mobile Platform
}

\author{
Moumita Majumder \\ Assistant Professor \\ NIT, Agartala \\ Tripura
}

\author{
Sumit Dhar \\ IT Analyst \\ TCS, Kolkata \\ West Bengal
}

\author{
Subrata Debbarma \\ M.Tech Student \\ Department of CSE \\ Tripura University
}

\author{
P.N.Basu \\ Professor \\ Deptt. of SET \\ Jadavpur University
}

\begin{abstract}
A Content browsing through mobile requires that contents must fit on to mobile screen and it should be readable. A vast set of documents are already available that are designed for browsing with desktop computers. Problem arises when one wants to browse these documents with handheld devices e.g. mobile, PDAs, palm computers etc. A Mobile device with small display screen size is capable of showing 3 to 4 rows per screen and not more than 20 characters per line. Usability problem occurs if too much scrolling has to be done while browsing the document. This has brought out an immediate requirement of a search for some typical format to be followed while adapting content in order to display the same meaningfully on small screen. Also an experimental exploration is needed to find out conversion technologies for displaying content comfortably on mobile so that user can read it without any irritation. This paper explores some document analysis techniques and represents a technique using J2ME that could ease content browsing for mobile users. Present work analyzes text contents only.
\end{abstract}

\section{General Terms}

Mobile platform, Adaptation, Standalone content, J2ME.

\section{Keywords}

Keywords are your own designated keywords which can be used for easy location of the manuscript using any search engines.

\section{INTRODUCTION}

Web document manipulation for small screen devices is an important and active research domain today. However standalone documents are available in large quantities and in different forms. Such documents may be classified as

1) Simple Text Documents

2) Multimedia based Documents

The concept of accessing a standalone computer based document is aimed at desktop devices. With a phenomenal increase in the number of mobile phone usage and due to the fact that learning via mobile devices is an inescapable part of today's life, the necessity to bring the standalone content processing and adaptation tool for the mobile devices can hardly be overstressed. Comfortable visualization of traditional documents on these small screens is anything but practical [1]. Here, the present study addresses the challenge of improving the quality of information representation for mobile devices in case of accessing standalone learning content, since the hardware support on the mobile device is limited. Once standalone content such as notepad file is downloaded into the mobile device, there is a need to process the file content so that it can be represented in required format in multiple frames keeping in mind the small display area available. This paper reports a technique for adapting existing contents into mobile platform prepared for viewing on large display.

\section{RELATED WORK}

Handhelds are gaining popularity in web browsing application with the expansion of wireless internet. Traditional web content are designed to browse with desktop devices. With the growing demand of browsing the same on mobile, researchers already have started working on this area and developed several techniques to fit the web content on mobile. A review work by Author Hasan Alam and Faud Rahman [2] analyzes some techniques to manipulate contents for small screen display, research work by Virpi Roto [3] discusses some layouts to ease browsing and work by author Yevgen Borodin [4] developed a Model- directed Web transactional system for handhelds to ease browsing.

A study at Indian Institute of Technology, Delhi focuses on adaptation of Internet Services to wireless devices. Along with translation of HTML document to WML, they have divided the web pages to different relevant sub-sections after thorough analysis of the original web page. The client requests for a URL and the HTML page is fetched and passed to adaptation system. This system uses adaptation rules to select content and transcode it to WML. The output is a set of WML decks corresponding to requested HTML page. The content adaptation system aims to provide a transparent view of the HTML page through this set of WML decks [5].

All these works have put light on the problem to some extent. Some ways by which web pages can be manipulated are outlined namely Transcoding [6], Automated Re-authoring [7], Table of Content [8], Summarization [9] etc. These works have no doubt created a new era for mobile users as web browsing with Handhelds is no more a dream. It is practical and possible. For 
comfortable visualization Oracle9iAS Developers Guide developed XML Version 1 to create new contents for mobile [10]. This language works efficiently on a Mobile Simulator called Openwave Simulator. However comfortable visualization of standalone contents on Mobile is still on demand.

\section{DISSEMINATION ARCHITECTURE}

There are three parts of the present content adaptation process.(Fig 1)

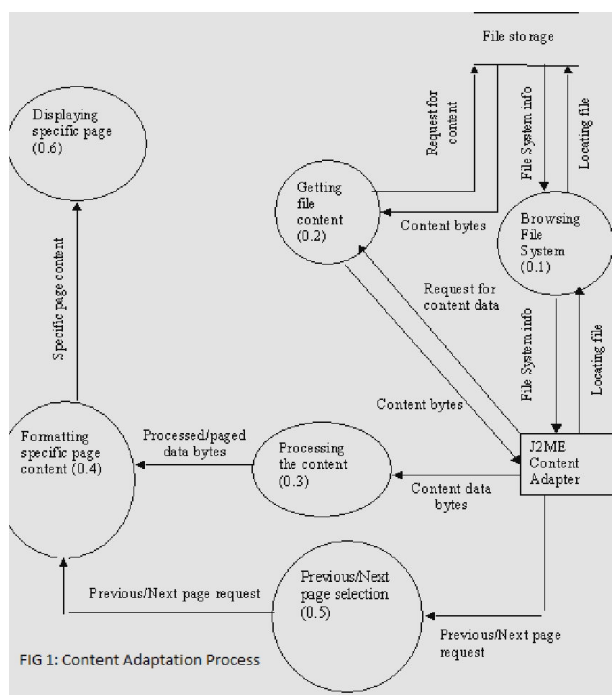

FIG 1: Content

Adaptation Process

\subsection{Locating Downloading File}

The adaptation tool provides the most important feature of file browsing. The initial functionality will browse through the file system of the mobile device and locate the required file.(Fig 2)

\section{FIG 2: File Navigation}

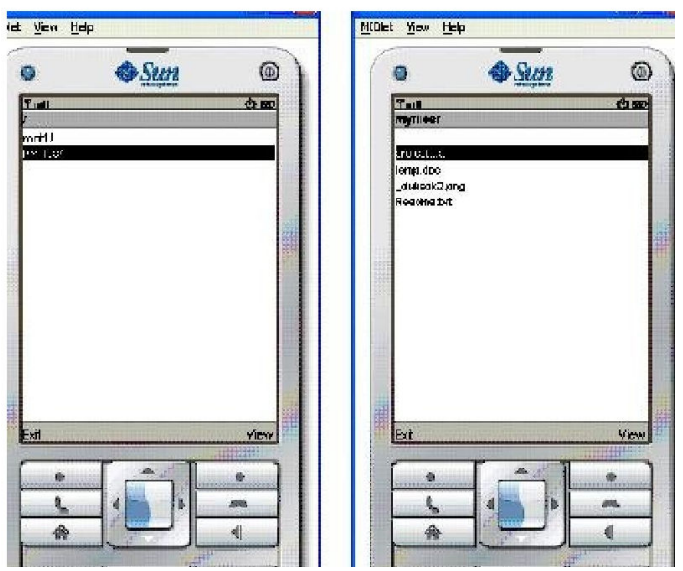

\subsection{Processing the Content}

Next functionality performed by the adaptation tool is processing the file content to make it presentable on the smaller display area of the mobile device. For that, the tool divides the file content into pages with smaller no of data bytes. This tool also adjusts the alignment of the text by removing unnecessary line breaks but maintaining the required paragraph breaks.

\subsection{Displaying Page With Proper Formatting}

The final functionality of the tool is to display the content divided into pages on the screen. The user can move through next or previous pages using proper menus by pressing corresponding keys of the mobile device. There are other user-friendly menus for closing the current file on display, moving up the file/folder hierarchy, closing the application as a whole etc.(Fig 3)
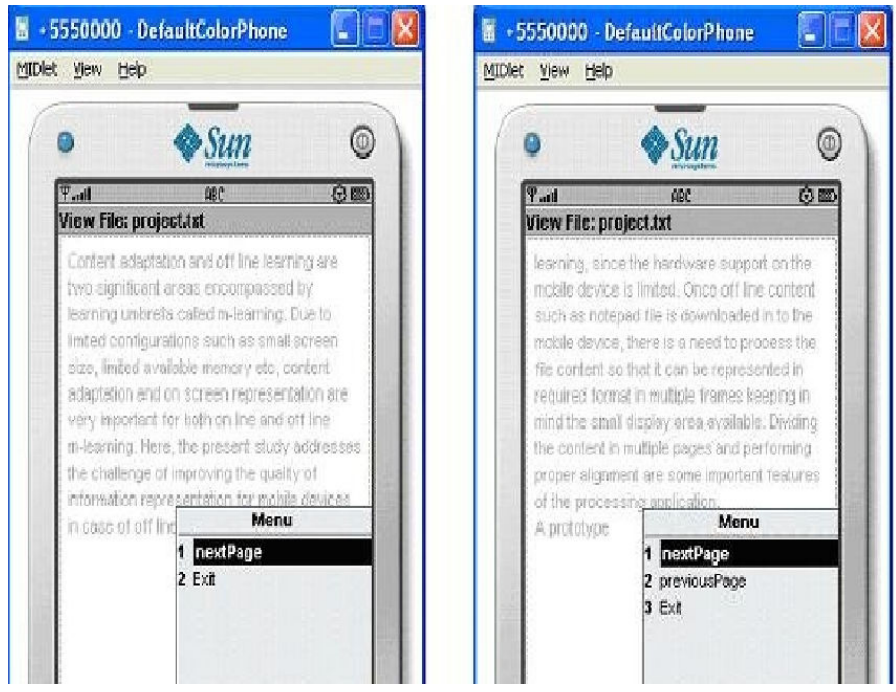

FIG 3 Navigating through Formatted page

\section{IMPLEMENTATIONS}

The main aim of the present work is to design and develop a technique to adapt standalone text content for mobile platform. As the platform is mobile so, the size of deployable and resource requirement during execution should be minimum. Keeping this in mind, it can be easily identified that most of the mobile device vendors support JAVA enabled applications. So, if J2ME is used then other dependable are need not to be installed. This dissemination needs File Connection Optional package. This comes with J2ME.

Fig 3 shows a simulated environment after implementation. Once the notepad file is downloaded into the mobile device, that file can be located using the adapter tool. All the file system roots and subfolder locations can be explored using this tool. Then the file is opened. Next the tool processes file content and first of all divides the whole content into sub frames, which will be displayed as individual pages. The next page/previous page menu options will be available as required so that the user can navigate through various pages as intended by him.

\section{LIMITATIONS AND FUTURE WORK}

This paper simulated the technology only for Text based standalone content. Multimedia based contents are put aside for future work. Discarding irrelevant contents from a document and then displaying the rest of the document is also kept for future work. Content analysis of Multimedia documents and deve lopment techniques to display the same on small screen is a challenging area of future work. 


\section{CONCLUSIONS}

Comfortable visualization of standalone content on mobile is a growing demand. But small memory, absence of secondary storage, small display screen has created problem for content browsing on Mobile. The aim of present work is to analyze the problem by analyzing previous work done on this area and present some approaches for converting standalone text content on small display for readable visualization. It has been observed that if comfortable visualization of standalone content can be made then it adds an academic value to a content browsing. User becomes capable of browsing specific content while on move.

\section{REFERENCES}

[1] T.F. Abdelzaher and N.Bhatti. Web Server Qos Management by Adaptive Content Delivery. Computer Networks, Elsevier, 31(11-16), 1999, PP 1563-1577

[2] Hassan Alam and Faud Rahman. Web Document Manipulation for Small Screen Devices: A Review. BCL Technologies Inc.

[3] Virpi Roto. Browsing on Mobile Phone. Nokia Research Center P.O.Box 407, 00045 NokiaGroup Finland

[4] Jalal Mahmud, yegen Borodin and I.V. Ramakrishnan. Content Analysis Techniques to ease Browsing with Handhelds. Department of Computer science, Stony Brook University, Stony Brook, NY 11794, USA
[5] Bringing Interner Services to Wireless Devices. Lipika Dey,B Raghu,Hitesh Sharma , Amit Sarma Department of Mathematics, Indian Institute of Science, Delhi.

[6] Y.Hwang, C. Jung, J. Kim, and S. Chung. WebAlchemist: A Web transcoding System for mobile Web Access in Handheld Devices. Proceedings of the Mobile Computing data Management, Denver, Colorado, August 2001

[7] T. bickmore, A. Girgensohn and J.W. Sullivan. Web page filtering and re-authoring for mobile users. The Computer Journal, 42(6), Oxford University Journal, 1999, pp. 534-546

[8] M. jones, g. Marsden, N. Mohd-Nasir, and G. Buchanan. A site based outliner for small screen Web access. Proceedings of the 8th World Wide Web conference, pages 156-157, 1999.

[9]. Buyukkokten, H. Garcia-Molina and A. Paepcke. Text Summarization of web pages on Handheld devices. Proceedings of the 2nd Meeting of the North American chapter of the association for Computational linguistics (NAACL), Pittsburgh, PA, U.S.A, 2-7 June, 2001

[10]Oracle9iAS Wireless Developer's Guide Release 2(9.0.2). http://download oracle.com/docs/cd/A97329 03/wireless.902/a90485/xml 2.htm 